\title{
What Is the Difference in Characteristics Between Belching Disorder and Gastroesophageal Reflux Disease With Belching? Centering Around Supragastric Belching
}

\author{
Sung Eun Kim \\ Department of Internal Medicine, Kosin University College of Medicine, Busan, Korea
}

Article: Characteristics of symptomatic belching in patients with belching disorder and patients who exhibit gastroesophageal reflux disease with belching

Jeong SO, Lee JS, Lee TH, et al

(J Neurogastroenterol Motil 2021;27:231-239)

Belching is an audible emission of air from the stomach or the esophagus through the pharynx. ${ }^{1}$ An occasional belching during or after meals is a normal physiological process; usually, belching up to 30 times a day is considered as a normal physiological venting mechanism. ${ }^{2}$ However, it becomes bothersome when it is too frequent to a point of affecting an individual's quality of life, and in such cases, medical attention is required. ${ }^{3}$ Unfortunately, since belching is a natural physiological phenomenon, excessive belching, which could be a belching disorder (BD), is rarely recognized as a significant health concern.

According to the Rome IV criteria, diagnosis of $\mathrm{BD}$ stands on the basis of in-depth medical history evaluation, and the diagnostic criterion is as follows; bothersome, which means severe enough to impair daily life activities, and belching from the esophagus or the stomach more than 3 days a week. Belching is further divided into excessive supragastric belching (SGB) which occurs in the esophagus and excessive gastric belching which occurs in the stomach. ${ }^{1}$ In terms of the clinical features, there was no established clinical correlation with gastric belching, however SGB is characterized by frequent, repetitive belching.

Belching is often associated with other disorders, especially gastrointestinal disorders. Gastrointestinal conditions that are accompanied by belching include rumination, gastroesophageal reflux disease (GERD), aerophagia, globus, and after antireflux surgery. ${ }^{4}$ Among them, belching is a relatively common symptom in GERD, a very common gastrointestinal disease. However, the prevalence of belching in GERD patients varies widely, 4.1-75.6\%. A Dutch study revealed that the incidence of SGB was significantly higher in GERD patients with severe belching compared to GERD patients with mild to moderate belching, therefore, they suggested that SGB, and not gastric belching influence the reflux symptoms in patients with GERD. ${ }^{6}$ A recent cross-cultural study reported that the prevalence of SGB and its effect on GERD symptoms is significantly lower in Japanese patients compared to British patients. Overall, research studies on belching in GERD patients are limited, with the majority of studies focusing on belching treatment.

Received: February 13, 2021 Revised: None Accepted: March 10, 2021

(.) This is an Open Access article distributed under the terms of the Creative Commons Attribution Non-Commercial License (http://creativecommons. org/licenses/by-nc/4.0) which permits unrestricted non-commercial use, distribution, and reproduction in any medium, provided the original work is properly cited.

*Correspondence: Sung Eun Kim, MD, PhD

Department of Internal Medicine, Kosin University College of Medicine, 262 Gamcheon-ro, Seo-gu, Busan 49267, Korea Tel: +82-51-990-5205, Fax: +82-51-990-5055, E-mail: solefide@hanmail.net 
In this issue of Journal of Neurogastroenterology and Motility, Jeong et $\mathrm{al}^{8}$ compared patients with $\mathrm{BD}(\mathrm{n}=10)$ and GERD $(\mathrm{n}=10)$ patients with belching, in a retrospective study that evaluated the characteristics of symptomatic belching. Symptomatic belching was analyzed according to the frequency, type, and acidity using multichannel intraluminal impedance and $\mathrm{pH}$ (MII$\mathrm{pH})$ monitoring with high-resolution manometry. Based on the study results, the number of symptomatic belching events in $\mathrm{BD}$ patients was higher than in GERD patients with belching (median: 160.5 vs $56.0, P<0.05$ ). The most frequent belching type in both groups was $\mathrm{SGB}$, and there was no significant difference in the ratio of belching type between $\mathrm{BD}$ and GERD patients (median: 93.0\% vs $86.8 \%$ ). However, the incidence rates of reflux-related SGB and preceding belching (which was defined as a belch that occurred immediately before the reflux event onset and evidenced as a $>50 \%$ reduction in the impedance level) were higher in GERD patients than patients with $\mathrm{BD}$ (median; reflux-related SGB: $78.3 \%$ vs 45.2\%, $P<0.005$; preceding belching: $31.8 \%$ vs $8.6 \%, P<$ 0.05 ). Regardless of SGB or gastric belching, acidic belching was significantly higher in GERD patients than $\mathrm{BD}$ patients (median; SGB: $38.1 \%$ vs $8.9 \%, P<0.05$; gastric belching: $49.2 \%$ vs $16.9 \%$, $P<0.05$ ). The number of SGB positively correlated with all reflux episodes in GERD patients (adjust $R^{2}=0.572, P=0.007$ ), while there was no correlation between them in patients with $\mathrm{BD}$.

The obvious limitations in this study are the retrospective study design and the small sample size. In addition, like in similar studies, MII-pH data was manually analyzed, therefore bias could have occurred during the interpretation.

Thus far, $\mathrm{BD}$ in the Asian population is still not clearly elucidated, there are few studies on BD and GERD patients with belching that have been conducted on an Asian population and especially in Korean patients. A recent review reported that the prevalence of belching in the general population varies from $6.7-28.8 \%,{ }^{5}$ however most of the studies were conducted in the Western nations.

In this study, authors classified SGB related to gastroesophageal reflux into preceding, during (which was defined as a belch that occurred during the reflux event), and following (which was defined as a belch that occurred immediately after the reflux event $[<1$ second $]$ ). Hemmink et al ${ }^{9}$ also reported 2 different categories of SGB in GERD patients. One was prior ( $<1$ second) to reflux and the other was during reflux, at proportions of $30 \%$ and $18 \%$, respectively. Notable is that Jeong et $\mathrm{al}^{8}$ suggested new patterns of
SGB; during-preceding belching, and during-isolated belching. There are interesting findings in the interpretation of the MII$\mathrm{pH}$ data, however, further study is needed on what these mean for belching patients. In addition, validation studies and clinical application using these findings will be required.

To the best of the author's knowledge, this is the first study to investigate the characteristics of symptomatic belching in BD and GERD patients with belching and to compare the differences in the 2 groups. Additionally, it is the first study on BD features in Korean BD patients. Further large-scale prospective studies are needed to understand the pathophysiology of belching in BD and GERD patients in the Asian population.

\section{Financial support: None.}

Conflicts of interest: None.

\section{References}

1. Stanghellini V, Chan FK, Hasler WL, et al. Gastroduodenal disorders. Gastroenterology 2016;150:1380-1392.

2. Bredenoord AJ, Weusten BL, Timmer R, Smout AJ. Air swallowing, belching, and reflux in patients with gastroesophageal reflux disease. Am J Gastroenterol 2006;101:1721-1726.

3. Kessing BF, Bredenoord AJ, Smout AJ. The pathophysiology, diagnosis and treatment of excessive belching symptoms. Am J Gastroenterol 2014;109:1196-1203.

4. Ooi JL, Vardar R, Sifrim D. Supragastric belching. Curr Opin Gastroenterol 2016;32:302-309.

5. Sawada A, Fujiwara Y, Sifrim D. Belching in gastroesophageal reflux disease: literature review. J Clin Med 2020;9:3360-3373.

6. Kessing BF, Bredenoord AJ, Velosa M, Smout AJ. Supragastric belches are the main determinants of troublesome belching symptoms in patients with gastro-oesophageal reflux disease. Aliment Pharmacol Ther 2012;35:1073-1079.

7. Sawada A, Itami H, Nakagawa K, et al. Supragastric belching in Japan: lower prevalence and relevance for management of gastroesophageal reflux disease compared to United Kingdom. J Gastroenterol 2020;55:1046-1053.

8. Jeong SO, Lee JS, Lee TH, et al. Characteristics of symptomatic belching in patients with belching disorder and patients who exhibit gastroesophageal reflux disease with belching. J Neurogastroenterol Motil 2021;27:231-239.

9. Hemmink GJ, Bredenoord AJ, Weusten BL, Timmer R, Smout AJ. Supragastric belching in patients with reflux symptoms. Am J Gastroenterol 2009;104:1992-1997. 